\title{
Front Matter: Volume 7503
}

, "Front Matter: Volume 7503," Proc. SPIE 7503, 20th International Conference on Optical Fibre Sensors, 750301 (5 October 2009); doi: 10.1117/12.846061

SDIE Event: 20th International Conference on Optical Fibre Sensors, 2009, SPIE. Edinburgh, United Kingdom 


\title{
PROCEEDINGS OF SPIE
}

\section{0th International Conference on Optical Fibre Sensors}

\author{
Julian Jones \\ Brian Culshaw \\ Wolfgang Ecke \\ José Miguel López-Higuera \\ Reinhardt Willsch \\ Editors \\ 5-9 October 2009 \\ Edinburgh, Scotland, United Kingdom \\ Organised by \\ IOP-Institute of Physics (United Kingdom) \\ Cooperating Organisations \\ SPIE \\ Optical Society of America \\ Published by \\ SPIE
}

Part One of Two Parts

Volume 7503 
The papers included in this volume were part of the technical conference cited on the cover and title page. Papers were selected and subject to review by the editors and conference program committee. Some conference presentations may not be available for publication. The papers published in these proceedings reflect the work and thoughts of the authors and are published herein as submitted. The publisher is not responsible for the validity of the information or for any outcomes resulting from reliance thereon.

Please use the following format to cite material from this book:

Author(s), "Title of Paper," in 20th International Conference on Optical Fibre Sensors, edited by Julian Jones, Brian Culshaw, Wolfgang Ecke, José Miguel López-Higuera, Reinhardt Willsch, Proceedings of SPIE Vol. 7503 (SPIE, Bellingham, WA, 2009) Article CID Number.

ISSN 0277-786X

ISBN 9780819478146

Published by

SPIE

P.O. Box 10, Bellingham, Washington 98227-0010 USA

Telephone +1 3606763290 (Pacific Time) · Fax +1 3606471445

SPIE.org

Copyright (C) 2009, Society of Photo-Optical Instrumentation Engineers

Copying of material in this book for internal or personal use, or for the internal or personal use of specific clients, beyond the fair use provisions granted by the U.S. Copyright Law is authorized by SPIE subject to payment of copying fees. The Transactional Reporting Service base fee for this volume is $\$ 18.00$ per article (or portion thereof), which should be paid directly to the Copyright Clearance Center (CCC), 222 Rosewood Drive, Danvers, MA 01923. Payment may also be made electronically through CCC Online at copyright.com. Other copying for republication, resale, advertising or promotion, or any form of systematic or multiple reproduction of any material in this book is prohibited except with permission in writing from the publisher. The CCC fee code is 0277-786X/09/\$18.00.

Printed in the United States.

Publication of record for individual papers is online in the SPIE Digital Library.

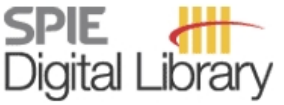

SPIEDigitalLibrary.org

Paper Numbering: Proceedings of SPIE follow an e-First publication model, with papers published first online and then in print and on CD-ROM. Papers are published as they are submitted and meet publication criteria. A unique, consistent, permanent citation identifier (CID) number is assigned to each article at the time of the first publication. Utilization of CIDs allows articles to be fully citable as soon they are published online, and connects the same identifier to all online, print, and electronic versions of the publication. SPIE uses a six-digit CID article numbering system in which:

- The first four digits correspond to the SPIE volume number.

- The last two digits indicate publication order within the volume using a Base 36 numbering system employing both numerals and letters. These two-number sets start with 00, 01, 02, 03, 04 , 05, 06, 07, 08, 09, OA, OB ... 0Z, followed by 10-1Z, 20-2Z, etc.

The CID number appears on each page of the manuscript. The complete citation is used on the first page, and an abbreviated version on subsequent pages. Numbers in the index correspond to the last two digits of the six-digit CID number. 


\title{
Contents
}

\section{Part One}

\author{
xxxi Conference Committees \\ xxxv Introduction
}

SPECIAL WORKSHOP SESSION ON PHOTONIC CRYSTAL FIBRES FOR SENSING I

750302 Coherent backscattering noise in a photonic-bandgap fiber optic gyroscope (Invited Paper) [7503-01]

M. J. F. Digonnet, S. W. Lloyd, S. Fan, Stanford Univ. (United States)

750303 Microstructured optical fibers for gas sensing: design, fabrication, and post-fab processing (Invited Paper) [7503-05]

M. N. Petrovich, C. Grivas, F. Poletti, A. van Brakel, E. Austin, D. J. Richardson, Univ. of Southampton (United Kingdom)

$750304 \quad$ Novel sensors based on hollow-core photonic bandgap fibers (Invited Paper) [7503-06] W. Jin, The Hong Kong Polytechnic Univ. (Hong Kong, China)

\section{SPECIAL WORKSHOP SESSION ON PHOTONIC CRYSTAL FIBRES FOR SENSING II}

750308 Nanofilm-based optical fiber sensor schemes (Invited Paper) [7503-02]

I. R. Matias, F. J. Arregui, I. del Villar, Univ. Pública de Navarra (Spain); R. O. Claus, Nanosonic, Inc. (United States)

\section{SMART STRUCTURES AND MATERIALS}

7503 OB Photonic skins for optical sensing: highlights of the PHOSFOS Project (Invited Paper) [7503-08]

F. Berghmans, H. Thienpont, Vrije Univ. Brussel (Belgium); P. Van Daele, IMEC (Belgium);

P. Dubruel, Univ. Gent (Belgium); W. Urbanczyk, Wroclaw Univ. of Technology (Poland);

J. Rayss, Maria Curie-Skłodowska Univ. (Poland); D. J. Webb, Aston Univ. (United Kingdom);

J. Vlekken, FOS\&S (Belgium); K. Kalli, Cyprus Univ. of Technology (Cyprus); K. Sugden,

Astasense Ltd. (United Kingdom)

7503 OC Distributed transverse load sensing in polarisation maintaining fibres using Rayleigh backscatter [7503-09]

R. R. J. Maier, W. N. MacPherson, J. S. Barton, Heriot-Watt Univ. (United Kingdom)

7503 OD Distributed strain measurement with millimeter-order spatial resolution based on Brillouin optical correlation-domain reflectometry using tellurite glass fiber [7503-10]

Y. Mizuno, Z. He, K. Hotate, The Univ. of Tokyo (Japan)

$7503 \mathrm{OE}$ Application of FBG sensors in strengthening and maintenance monitoring of old bridges [7503-11]

L. Yue, J. Huang, Y. Yang, Wuhan Univ. of Technology (China) 
7503 OF Fibre Bragg grating based effective soil pressure sensor for geotechnical applications [7503-12]

R. Correia, J. Li, S. Staines, E. Chehura, S. W. James, Cranfield Univ. (United Kingdom); J. Kutner, P. Dewhurst, P. Ferreira, Univ. College London (United Kingdom); R. P. Tatam, Cranfield Univ. (United Kingdom)

7503 OG Dynamic, full-spectral interrogation of fiber Bragg grating sensors for impact testing of composite laminates [7503-13]

A. Propst, K. Peters, M. A. Zikry, North Carolina State Univ. (United States); W. Kunzler, Z. Zhu, M. Wirthlin, R. Selfridge, S. Schultz, Brigham Young Univ. (United States)

JOINT SESSION S\&A/OFS-20

$7503 \mathrm{OH} \quad$ Hazard monitoring in mines using fibre optic sensors (Invited Paper) [7503-14]

T. Liu, C. Wang, Y. Wei, Y. Zhao, Shandong Key Lab. of Fibre Optic Sensors (China); D. Huo, Shandong Micro-Sensor Photonics, Ltd. (China); Y. Shang, Z. Wang, Shandong Key Lab. of Fibre Optic Sensors (China); Y. Ning, Intelligent Sensor Systems Ltd. (United States)

7503 Ol Conception and preliminary evaluation of an optical fibre sensor for simultaneous measurement of pressure and temperature [7503-15]

K. Bremer, E. Lewis, B. Moss, G. Leen, S. Lochmann, Univ. of Limerick (Ireland); I. Mueller, Hochschule Wismar (Germany)

7503 0J Multi-zone temperature sensor using a multi-wavelength Brillouin fiber ring laser [7503-16] C. A. Galindez, F. J. Madruga, A. Ullan, Univ. de Cantabria (Spain); M. Lopez-Amo, Univ. Pública de Navarra (Spain); J. M. Lopez-Higuera, Univ. de Cantabria (Spain)

7503 OK Use of fibre sensors for temperature measurement in subsea infrastructure to monitor flow-loop cool-down [7503-17]

D. M. Faichnie, A. Graham, L. Costello, D. McStay, FMC Technologies Ltd. (United Kingdom)

$7503 \mathrm{OL}$ Optical fiber humidity sensor based on surface plasmon resonance in the infra-red region [7503-18]

M. Hernáez, C. R. Zamarreño, I. R. Matías, F. J. Arregui, Univ. Pública de Navarra (Spain)

$75030 \mathrm{M}$ Applications of polymer optical fibre grating sensors to condition monitoring of textiles [7503-19]

C. C. Ye, J. M. Dulieu-Barton, Univ. of Southampton (United Kingdom); D. J. Webb, C. Zhang, Aston Univ. (United Kingdom); G.-D. Peng, Univ. of New South Wales (Australia);

A. R. Chambers, F. J. Lennard, D. D. Eastop, Univ. of Southampton (United Kingdom) 
7503 ON Raman meets medicine: Raman spectroscopy: a powerful tool in biophotonics (Invited Paper) [7503-20]

J. Popp, Institut für Photonische Technologien Jena (Germany) and Friedrich-Schiller-Univ. Jena (Germany); M. Schmitt, Institut für Photonische Technologien Jena (Germany);

B. Dietzek, Institut für Photonische Technologien Jena (Germany) and Friedrich-Schiller-Univ. Jena (Germany); C. Krafft, Friedrich-Schiller-Univ. Jena (Germany); R. Möller, Institut für Photonische Technologien Jena (Germany) and Friedrich-Schiller-Univ. Jena (Germany); P. Rösch, Institut für Photonische Technologien Jena (Germany)

7503 OP Surface plasmon resonance biosensors: advances and applications (Invited Paper) [7503-23]

J. Homola, Institute of Photonics and Electronics (Czech Republic)

SPECIAL WORKSHOP SESSION ON BIOPHOTONIC INSTRUMENTATION AND SENSING II

7503 OQ Advanced biomedical optical fibre sensors (Invited Paper) [7503-24]

F. Baldini, Institute of Applied Physics, CNR (Italy)

POSTERS I

7503 OT Fiber optic gas detection system for health monitoring of oil-filled transformer [7503-26] H. L. Ho, J. Ju, W. Jin, The Hong Kong Polytechnic Univ. (Hong Kong, China)

7503 OU Comparison of surface functionalization processes for optical fibre biosensing applications [7503-27]

A. François, H. Ebendorff-Heidepriem, T. M. Monro, Univ. of Adelaide (Australia)

7503 OV Closely spaced fibre Bragg grating sensors for detailed measurement of peristalsis in the human gut [7503-28]

J. W. Arkwright, CSIRO (Australia); P. G. Dinning, St. George Hospital, Univ. of New South Wales (Australia); I. D. Underhill, S. A. Maunder, N. Blenman, CSIRO (Australia);

M. M. Szczesniak, I. J. Cook, St. George Hospital, Univ. of New South Wales (Australia)

7503 OW Optical fiber sensors based on indium tin oxide surface plasmon resonance supporting coatings [7503-29]

C. R. Zamarreño, M. Hernáez, I. R. Matías, F. J. Arregui, Univ. Pública de Navarra (Spain)

7503 OX Swept-source OCT with coherent imaging fibre bundles [7503-30]

H. D. Ford, R. P. Tatam, Cranfield Univ. (United Kingdom)

7503 OY Ultra-high sensitive sensitive hydrogen sensor using higher order cladding mode coupled by a palladium-coated long-period fiber grating [7503-31]

Y.-H. Kim, Gwangju Institute of Science and Technology (Korea, Republic of); M. J. Kim, B. S. Rho, Korea Photonics Technology Institute (Korea, Republic of); H. Y. Choi, M. S. Park, J.-H. Jang, B.-H. Lee, Gwangju Institute of Science and Technology (Korea, Republic of) 
$75030 Z$ Tuneable ring-DFB PCF laser for ultra-high resolution spectroscopy [7503-32] K. Cook, J. Canning, C. K. Poon, Univ. of Sydney (Australia); A. A. P. Pohl, Federal Univ. of Technology (Brazil)

750310 Evaluation and calibration of FBG-based relative humidity sensor designed for structural health monitoring [7503-33]

T. Venugopalan, T. L. Yeo, City Univ. London (United Kingdom); F. Basedau, A. S. Henke, Bundesanstalt für Materialforschung und -prüfung (Germany); T. Sun, City Univ. London (United Kingdom); K. T. V. Grattan, City Univ., London (Germany); W. Habel, Bundesanstalt für Materialforschung und -prüfung (Germany)

750311 Surface plasmon resonance fiber sensor for in situ monitoring of the deposition of $\mathrm{nm}$-scale polymer films [7503-34]

Y. Shevchenko, N. U. Ahamad, G. Galway, A. lanoul, J. Albert, Carleton Univ. (Canada)

750312 Intra-cavity spectroscopy using amplified spontaneous emission in erbium fibre lasers [7503-35]

N. Arsad, Univ. of Strathclyde (United Kingdom) and National Univ. of Malaysia (Malaysia);

G. Stewart, Univ. of Strathclyde (United Kingdom)

750313 Recent advance in tunable diode laser spectroscopy with background RAM nulling for industrial applications [7503-36]

K. Ruxton, A. L. Chakraborty, A. J. McGettrick, K. Duffin, W. Johnstone, G. Stewart, Univ. of Strathclyde (United Kingdom)

750314 Fibre optic chloride sensor based on fluorescence quenching of an acridinium dye [7503-37]

T. H. Nguyen, City Univ. London (United Kingdom); Y. C. Lin, C. T. Chen, National Taiwan Univ. (Taiwan); F. Surre, T. Venugopalan, T. Sun, K. T. V. Grattan, City Univ. London (United Kingdom)

750315 Polarization properties of fiber cladding modes near cut-off with applications to refractometry [7503-38]

F. Gorlier, C. Caucheteur, M. Wuilpart, P. Mégret, Faculté Polytechnique de Mons (Belgium);

J. Albert, Carleton Univ. (Canada)

750316 Remote sensing network to detect and identify organic vapours [7503-39]

C. Elosua, R. A. Perez-Herrera, M. Lopez-Amo, C. Bariain, Univ. Pública de Navarra (Spain);

A. Luquin, M. Laguna, Univ. de Zaragoza (Spain)

750317 All-fiber low-temperature hydrogen sensing using a multi-functional light source [7503-40] T. Chen, M. P. Buric, D. Xu, K. P. Chen, Univ. of Pittsburgh (United States); P. R. Swinehart, M. Maklad, Lake Shore Cryotronics (United States)

750318 Plastic fibre optic oxygen sensors based on polymer matrix doped with Pt (II) complexes [7503-41]

R. Chen, A. D. Farmery, Oxford Univ. (United Kingdom); A. N. Obeid, Oxford Optronix (United Kingdom); C. E. W. Hahn, Oxford Univ. (United Kingdom) 
750319 An infrared fibre evanescent wave spectroscopic (FEWS) sensor using purified GeTeSe chalcogenide fibres [7503-42]

X. Jiang, A. Jha, Univ. of Leeds (United Kingdom)

$75031 \mathrm{~A}$ Interferometric fibre-optic sensor for acetic acid measurement [7503-43]

C. Jesus, INESC Porto (Portugal) and Univ. of Madeira (Portugal); S. F. O. Silva, INESC Porto (Portugal) and Univ. do Porto (Portugal); M. Castanheira, G. Gonzalez Aguilar, INESC Porto (Portugal); O. Frazão, INESC Porto (Portugal) and Univ. do Porto (Portugal); P. A. S. Jorge, INESC Porto (Portugal); J. M. Baptista, INESC Porto (Portugal) and Univ. da Madeira (Portugal)

7503 1B Fibre Bragg gratings as interrogation elements for surface plasmon resonance sensors [7503-44]

N. Díaz-Herrera, Univ. Complutense de Madrid (Spain); D. Viegas, Univ. Do Porto (Portugal) and INESC Porto (Portugal); P. A. S. Jorge, INESC Porto (Portugal); M. C. Navarrete, Univ. Complutense de Madrid (Spain); L. A. Ferreira, F. M. Araújo, INESC Porto (Portugal) J. L. Santos, Univ. Do Porto (Portugal) and INESC Porto (Portugal); A. González-Cano, Univ. Complutense de Madrid (Spain)

7503 1C Packaged symmetric/asymmetric corrugated long period fiber gratings for refractive index sensing applications [7503-45]

C.-Y. Huang, W.-L. Chan, S.-M. Chuo, J.-H. Chang, L.-L. Chen, L. A. Wang, National Taiwan Univ. (Taiwan)

7503 1D Highly sensitive and linear optical fiber carbon dioxide sensor based on sol-gel matrix doped with silica particles and HPTS [7503-46]

Y.-L. Lo, C.-S. Chu, National Cheng Kung Univ. (Taiwan)

7503 1E Automatic multiple chemical components distinguishment [7503-47] N. Ni, C. C. Chan, P. Shum, Nanyang Technological Univ. (Singapore)

7503 IF A statically scanned tandem interferometer [7503-48]

D. F. Murphy, D. A. Flavin, Waterford Institute of Technology (Ireland)

7503 1G Long period gratings working in transition mode as a valuable technological platform for biosensing [7503-49]

P. Pilla, Univ. degli Studi del Sannio (Italy); P. Foglia Manzillo, Univ. of Naples, Parthenope (Italy); V. Malachovska, Institute of Composite and Biomedical Materials, CNR (Italy); S. Campopiano, Univ. of Naples, Parthenope (Italy); A. Cutolo, Univ. degli Studi del Sannio (Italy); M. Giordano, Institute of Composite and Biomedical Materials, CNR (Italy);

A. Cusano, Univ. degli Studi del Sannio (Italy)

$75031 \mathrm{H} \quad$ Characteristics of hetero-core spliced fiber optics in simultaneous utilization of humidity sensing and data communications [7503-50]

N. Shinomiya, E. Kondo, S. Akita, M. Nishiyama, H. Sasaki, K. Watanabe, Soka Univ. (Japan)

$750311 \quad$ Species selective vapour sensing of organic compounds using dual resonant long period fibre gratings with a calixarene coating [7503-51]

S. M. Topliss, S. W. James, F. Davis, S. P. J. Higson, R. P. Tatam, Cranfield Univ. (United Kingdom) 
$75031 \mathrm{~J}$ Wide range refractive index sensor using a twin-grating interferometer for intensity reference [7503-52]

M. G. Shlyagin, R. Martińez Manuel, Ctr. de Investigación Científica y de Educación Superior de Ensenada (Mexico); O. Esteban, Univ. de Alcalá de Henares (Spain)

7503 IK Photochemical properties of porphyrin films covering surfaces of tapered optical fibers [7503-53]

A. Veselov, C. Thür, V. Chukharev, M. Guina, H. Lemmetyinen, N. Tkachenko, Tampere Univ. of Technology (Finland)

7503 IL Plastic optical fiber sensor with humidity calibration for detecting propane and methane gas [7503-54]

M. Morisawa, N. Tanaike, S. Honma, S. Muto, Univ. of Yamanashi (Japan)

$75031 \mathrm{M}$ Subsurface imaging and cell refractometry using quantitative phase/shear-force feedback microscopy [7503-55]

K. Edward, F. Farahi, The Univ. of North Carolina at Charlotte (United States)

7503 iN Development and evaluation of a photometric fibre-optic sensor for monitoring abdominal organ photoplethysmographs and blood oxygen saturation [7503-56]

M. Hickey, City Univ. London (United Kingdom); N. Samuels, N. Randive, R. M. Langford, St. Bartholomew's Hospital (United Kingdom); P. A. Kyriacou, City Univ. London (United Kingdom)

$750310 \quad$ Long-distance fiber-optic $\boldsymbol{\Phi}$-OTDR intrusion sensing system [7503-57]

Y.-J. Rao, Univ. of Electronic Science and Technology of China (China) and Chongaing Univ. (China); J. Luo, Z. Ran, J. Yue, X. Luo, Univ. of Electronic Science and Technology of China (China); Z. Zhou, Harbin Institute of Technology (China)

7503 IP A real-time event classification system for a fibre-optic perimeter intrusion detection system [7503-58]

S. S. Mahmoud, J. Katsifolis, Future Fibre Technologies Pty Ltd. (Australia)

7503 1Q Ultra-long distance (300km) fiber Bragg grating sensor system using hybrid EDF and Raman amplification [7503-59]

Y.-J. Rao, Univ. Of Electronic Science and Technology of China (China) and Chongaing Univ. (China); S. Feng, Univ. of Electronic Science and Technology of China (China);

Q. Jiang, Chongqing Univ. (China); Z.-L. Ran, Univ. of Electronic Science and Technology of China (China)

7503 IR Self-referencing model for electro-optical WDM fiber-optic intensity-based sensor network [7503-60]

D. S. Montero, C. Vázquez, Carlos III Univ. of Madrid (Spain); J. M. Baptista, INESC Porto (Portugal) and Univ. da Madeira (Portugal); J. L. Santos, INESC Porto (Portugal) and Univ. do Porto (Portugal)

7503 is Fiber Bragg grating hydrophone array using multi-wavelength laser: simultaneous multipoint underwater acoustic detection [7503-61]

S. Tanaka, A. Wada, N. Takahashi, National Defense Academy (Japan) 
7503 1T Simple tunable pulsed laser system for quasi-distributed sensors [7503-62]

C. C. Kato, A. M. B. Braga, A. S. Ribeiro, Catholic Univ. of Rio de Janeiro (Brazil);

L. C. G. Valente, Lupatech Monitoring Systems (Brazil); S. R. K. Morikawa, Petrobras Research Ctr. (Brazil)

$75031 \mathrm{U} \quad$ Multipoint refractive index sensor for liquids based on optical fiber Bragg-gratings [7503-63] H. K. Bal, F. Sidiroglou, S. F. Collins, Z. Brodzeli, Victoria Univ. (Australia)

$75031 \mathrm{~V}$ Intensity sensors multiplexing using a multiwavelength ring fiber laser with hybrid serial-tree configuration [7503-64]

D. Passaro, Univ. of Parma (Italy); M. Fernàndez, R. A. Pérez-Herrera, C. Elosúa, C. Bariáin, Univ. Pública de Navarra (Spain); S. Selleri, Univ. of Parma (Italy); M. Lopez-Amo, Univ. Pública de Navarra (Spain)

7503 IW High speed optical interrogator calibration technique [7503-65]

D. S. George, S. Hill, R. I. Howden, G. W. Smith, AWE Aldermaston (United Kingdom)

$75031 \mathrm{1X} \quad$ Multipoint real-time displacement sensing method employing multi-wavelength by intensity-based hetero-core fiber optics sensors [7503-66]

Y. Koyama, M. Nishiyama, K. Watanabe, Soka Univ. (Japan)

7503 1Y Switchable multi-wavelength erbium-doped fiber laser for remote sensing [7503-67] R. A. Perez-Herrera, S. Diaz, M. Fernández-Vallejo, M. Lopez-Amo, Univ. Pública de Navarra (Spain); M. A. Quintela, J. M. López-Higuera, Univ. de Cantabria (Spain)

750312 Multipoint measurement based on hetero-core structured optical fiber SPR sensor employing OTDR equipment [7503-68]

K. Takagi, M. Shiraishi, M. Nishiyama, A. Seki, K. Watanabe, Soka Univ. (Japan)

750320 Resilient long-distance sensor system using a multiwavelength Raman laser [7503-69] M. Fernandez-Vallejo, S. Díaz, R. A. Perez-Herrera, Public Univ. of Navarra (Spain); D. Passaro, S. Selleri, Univ. of Parma (Italy); M. A. Quintela, J. M. López Higuera, Univ. de Cantabria (Spain); M. Lopez-Amo, Public Univ. of Navarra (Spain)

$750321 \quad$ In-line Fabry-Perot sensors based on hollow-core photonic bandgap fibers for high temperature applications [7503-212]

Y.-J. Rao, Chongqing Univ. (China) and Univ. of Electronic Science \& Technology (China); M. Deng, Chongqing Univ. (China); T. Zhu, Chongqing Univ. (China) and Univ. of Electronic Science \& Technology (China); H. Li, Chongqing Univ. (China)

750322 Optical fibre spectroscopy sensor for the quantitative determination of industrial textile dyes [7503-280]

A. M. Cubillas, O. M. Conde, P. Anuarbe, Univ. de Cantabria (Spain); M. Gutierrez, V. Martinez, Textil Santanderina (Spain); J. M. Lopez-Higuera, Univ. de Cantabria (Spain)

750323 An extrinsic Fabry-Perot interferometric optical fiber sensor system for hydrogen detection [7503-281]

Z. Yang, M. Zhang, M. Lei, X. Yu, Y. Liao, S. Lai, Q. Tian, Tsinghua Univ. (China); Q. Li, Y. Zhang, Z. Zhuang, CAEP (China) 
750324 Rotational positioning measurement for the absolute angle based on a hetero-core fiber optics sensor [7503-211]

M. Nishiyama, K. Watanabe, Soka Univ. (Japan)

PHYSICAL, MECHANICAL, AND ELECTROMAGNETIC SENSORS

750326 Strong regenerated gratings [7503-71]

J. Canning, M. Stevenson, J. Fenton, M. Åslund, Univ. of Sydney (Australia);

S. Bandyopadhyay, Central Glass and Ceramic Research Institute (India)

750327 Optical bend sensor for vector curvature measurement based on Bragg grating in eccentric core polymer optical fibre [7503-94]

X. Chen, C. Zhang, D. J. Webb, R. Suo, Aston Univ. (United Kingdom); G. D. Peng, Univ. of New South Wales (Australia); K. Kalli, Higher Technical Institute (Cyprus)

750328 Use of Fabry-Pérot lasers for simultaneous distributed strain and temperature sensing based on hybrid Raman and Brillouin scattering [7503-73]

M. A. Soto, G. Bolognini, F. Di Pasquale, Scuola Superiore Sant'Anna (Italy)

750329 A fibre laser acoustic vector sensor [7503-74]

P. Jackson, S. Foster, S. Goodman, Defence Science and Technology Organisation (Australia)

$75032 \mathrm{~A} \quad$ Fabry-Perot cavity sensing structure based on a suspended-core fibre [7503-75] O. Frazão, INESC Porto (Portugal) and Univ. do Porto (Portugal); J. M. Baptista, INESC Porto (Portugal) and Univ. da Maderia (Portugal); J. L. Santos, INESC Porto (Portugal) and Univ. do Porto (Portugal); J. Kobelke, K. Schuster, Institut für Photonische Technologien Jena (Germany)

\section{DISTRIBUTED SENSING}

$75032 \mathrm{~B} \quad$ Long-distance remote sensors for simultaneous measurement of strain and temperature based on multiwavelength fiber lasers (Invited Paper) [7503-76]

Y.-G. Han, Hanyang Univ. (Korea, Republic of)

7503 2C Optimized configuration for high resolution distributed sensing using Brillouin echoes [7503-77]

S. Foaleng-Mafang, J.-C. Beugnot, L. Thévenaz, Ecole Polytechnique Fédérale de Lausanne (Switzerland)

7503 2D Frequency-shifted light storage in a photonics crystal fiber via stimulated Brillouin scattering [7503-78]

Y. Dong, X. Bao, W. Li, Y. Li, Univ. of Ottawa (Canada)

$75032 \mathrm{E} \quad$ Noise of long-range optical frequency domain reflectometry after optical source phase noise compensation [7503-79]

X. Fan, Y. Koshikiya, F. Ito, NTT Access Network Service Systems Labs. (Japan) 
$75032 \mathrm{~F}$ A novel signal restoration method in the frequency domain to enhance spatial resolution in distributed Brillouin sensing [7503-80]

N. Nöther, P. Lenke, K. Krebber, Bundesanstalt für Materialforschung und -prüfung (Germany)

$75032 \mathrm{G} \quad$ 12-km distributed fiber sensor based on differential pulse-width pair BOTDA [7503-81] Y. Dong, X. Bao, W. Li, Univ. of Ottawa (Canada)

\section{MULTIPLEXING AND SENSOR NETWORKING}

$75032 \mathrm{H} \quad$ Spectrally coded multiplexing for fibre grating sensor systems (Invited Paper) [7503-82] P. Childs, Univ. of New South Wales (Australia) and Tsinghua Univ. (China); A. C. L. Wong, Univ. of New South Wales (Australia); B. Yan, Univ. of New South Wales (Australia) and Beijing Univ. of Posts and Telecommunications (China); M. Li, Univ. of New South Wales (Australia) and Harbin Institute of Technology (China); G.-D. Peng, Univ. of New South Wales (Australia)

$750321 \quad$ All-fiber hybrid fiber Bragg grating cavity for multi-parameter sensing applications [7503-83] D. Paladino, G. Quero, Univ. of Sannio (Italy); A. Iadicicco, Univ. of Naples, Parthenope (Italy); C. Caucheteur, P. Mégret, Faculté Polytechnique de Mons (Belgium); A. Cusano, Univ. of Sannio (Italy)

$75032 \mathrm{~J} \quad$ Multiplexed long-length fiber Bragg grating distributed sensors based on synthesis of optical coherence function [7503-84]

K. Kajiwara, K. Hotate, The Univ. of Tokyo (Japan)

$75032 \mathrm{~K} \quad$ Ultra-short distributed Bragg reflector fiber lasers for sensing applications [7503-85] Y. Zhang, Dalian Univ. of Technology (China); B.-O. Guan, Jinan Univ. (China) and Dalian Univ. of Technology (China); H.-Y. Tam, The Hong Kong Polytechnic Univ. (Hong Kong, China)

$75032 \mathrm{~L} \quad$ Novel system to interrogate distributed fiber strain sensors and point temperature sensors based on pulse correlation and FBGs [7503-86]

A. Bueno, Univ. Politécnica de Valencia (Spain); K. Nonaka, Kochi Univ. of Technology (Japan); S. Sales, Univ. Politécnica de Valencia (Spain)

$75032 \mathrm{M} \quad$ A low-loss interferometric sensor array with unequal segments [7503-87] A. Isseroff, M. Tur, A. Eyal, Tel Aviv Univ. (Israel)

\section{POSTERS II}

$75032 \mathrm{~N} \quad$ Optical fibre Bragg gratings for high temperature sensing [7503-88]

J. Canning, M. Stevenson, K. Cook, M. Aslund, Univ. of Sydney (Australia); W. Ecke, R. Willsch, H. Bartelt, IPHT Jena (Germany); H. J. Kalinowski, L. Grabarski, V. Oliveira, Univ. Technologica Federal do Parana (Brazil); C. Martelli, A. Braga, Pontificia Univ. Católica do Rio de Janeiro (Brazil); N. Groothoff, G.-D. Peng, Univ. of New South Wales (Australia) 
750320 Sensing properties of germanate and tellurite glass optical fibres [7503-89]

H. Li, Heriot-Watt Univ. (United Kingdom); J. Lousteau, Univ. of Leeds (United Kingdom);

R. Suo, Aston Univ. (United Kingdom); X. Jiang, Univ. of Leeds (United Kingdom);

W. N. MacPherson, H. T. Bookey, J. S. Barton, A. K. Kar, Heriot-Watt Univ. (United Kingdom);

L. Zhang, Aston Univ. (United Kingdom); A. Jha, Univ. of Leeds (United Kingdom); I. Bennion,

Aston Univ. (United Kingdom)

$75032 \mathrm{P} \quad$ Dynamic strain sensor based on two wave mixing in optical fibers [7503-90]

S. Molin, I. Abada, D. Dolfi, J.-P. Huignard, Thales Research \& Technology (France); M. Doisy, Thales Underwater Systems (France)

$75032 Q \quad$ Temperature self-compensation method of hydrogen gas sensor based on a double cladding fiber interferometer [7503-91]

M. J. Kim, J. W. Lim, Korea Photonics Technology Institute (Korea, Republic of); Y. Kim,

B. H. Lee, Gwangju Institute of Science and Technology (Korea, Republic of); B. S. Rho,

Korea Photonics Technology Institute (Korea, Republic of)

$75032 R \quad$ Interferometric interrogation of a multicore fiber, two-axis inclinometer [7503-92]

G. A. Miller, C. G. Askins, Naval Research Lab. (United States); G. A. Cranch, SFA Inc. (United States)

750325 Simultaneous measurement of liquid level and temperature employing a single uniform FBG [7503-93]

X. Shu, K. Sugden, I. Bennion, Aston Univ. (United Kingdom)

$75032 \mathrm{~T}$ Passive, multiplexable measurement of magnetic field strength and temperature using PM fibre Bragg gratings and chiral fibre devices [7503-72]

P. Orr, P. Niewczas, Univ. of Strathclyde (United Kingdom)

$75032 \mathrm{U}$ An ultrahigh resolution FBG dynamic strain sensing system with eigenvector spectrum analysis method [7503-95]

Z.-L. Ran, Y.-J. Rao, J. Lu, Univ. of Electronics Science \& Technology (China)

$75032 V \quad$ Acoustic emission sensor based on biconical fiber micro-tapers [7503-96]

Y. Li, X. Wang, X. Bao, Univ. of Ottawa (Canada)

$75032 \mathrm{~W}$ Regeneration of fiber Bragg gratings in photosensitive fibers without hydrogen loading [7503-97]

E. Lindner, IPHT Jena (Germany); C. Chojetzki, FBGS Technologies GmbH (Germany);

S. Brückner, M. Becker, M. Rothhardt, H. Bartelt, IPHT Jena (Germany)

$75032 X \quad$ Laser-machined all-fiber in-line tip pressure sensor [7503-98]

Z. Ran, Univ. of Electronic Science and Technology of China (China); Y. Rao, Univ. of Electronic Science and Technology of China (China) and Chongqing Univ. (China); J. Zhang, B. XU, Univ. of Electronic Science and Technology of China (China) 
$75032 Y \quad$ Strain and temperature characterization of interferometric sensors based on ARROW fibers [7503-99]

S. H. Aref, INESC Porto (Portugal) and Univ. of Shahid Beheshti (Iran, Islamic Republic of);

O. Frazão, INESC Porto (Portugal) and Univ. do Porto (Portugal); P. Caldas, INESC Porto

(Portugal), Univ. do Porto (Portugal), and Escola superior de Tecnologia e Gestão de Viana do Castelo (Portugal); L. A. Ferreira, F. M. Araújo, INESC Porto (Portugal); J. L. Santos, INESC Porto (Portugal) and Univ. do Porto (Portugal); H. Latifi, Shahid Beheshti Univ. (Iran, Islamic Republic of); J. Ballato, Clemson Univ. (United States); T. Her, F. Farahi, The Univ. of North Carolina at Charlotte (United States)

$75032 Z$ Temperature and strain discrimination using a single fibre Bragg grating written in a tapered optical fibre [7503-100]

H. F. Lima, P. F. Antunes, Univ. de Aveiro (Portugal); R. N. Nogueira, Instituto de Telecomunicações (Portugal); J. L. Lemos Pinto, Univ. de Aveiro (Portugal)

750330 High mechanical-Q Lorentz force sensor for DC magnetic field measurement [7503-101] G. A. Cranch, C. G. Askins, R. E. Bartolo, G. A. Miller, C. K. Kirkendall, Naval Research Lab. (United States)

750331 Direct monitoring of underwater ultrasonic transducers in the near field using fibre Bragg grating sensors [7503-102]

G. M. H. Flockhart, M. McGuire, S. G. Pierce, G. Thursby, G. Stewart, G. Hayward, B. Culshaw, Univ. of Strathclyde (United Kingdom)

750332 Delayed transmission/reflection ratiometric reflectometry [7503-103]

S. Onoda, K. Inove, K. Aita, T. Nakada, M. Nakano, Y. Komatsu, Watanabe Co., Ltd. (Japan)

750333 Temperature independent bend sensor using Bragg gratings in Gemini fiber [7503-104] E. Zetterlund, A. Loriette, C. Sterner, M. Eriksson, H. Eriksson-Quist, W. Margulis, Acreo AB (Sweden)

750334 Pressure measurements on aircraft wing using phase-shifted fibre Bragg grating sensors [7503-105]

E. Chehura, S. W. James, N. Lawson, K. P. Garry, R. P. Tatam, Cranfield Univ. (United Kingdom)

750335 Tilted fiber Bragg grating-based accelerometer [7503-106]

T. Guo, L. Shao, H. Au, H.-Y. Tam, The Hong Kong Polytechnic Univ. (Hong Kong, China);

J. Albert, Carleton Univ. (Canada)

750336 Temperature and strain discriminating sensor based on the monitoring of cladding modes of a single femtosecond inscribed grating [7503-107]

N. Jovanovic, R. J. Williams, Macquarie Univ. (Australia); J. Thomas, Friedrich-Schiller-Univ. Jena (Germany); M. J. Steel, G. D. Marshall, A. Fuerbach, Macquarie Univ. (Australia); S. Nolte, A. Tünnermann, Friedrich-Schiller-Univ. Jena (Germany); M. J. Withford, Macquarie Univ. (Australia)

750337 Response of fiber Bragg grating transmission dips at twice the Bragg wavelength to transverse strain [7503-108]

H. K. Bal, F. Sidiroglou, S. P. Yam, Z. Brodzeli, Victoria Univ. (Australia); S. A. Wade, Monash

Univ. (Australia); G. W. Baxter, S. F. Collins, Victoria Univ. (Australia) 
750338 A fiber-optic CT using a reflection type Sagnac coil and a time-delayed sampling demodulation [7503-109]

H.-J. Park, H.-J. Kim, Y. H. Cho, M. Song, Chonbuk National Univ. (Korea, Republic of)

750339 Sensing characteristics of hollow-core photonic crystal fibre modal interferometers [7503-110]

S. H. Aref, INESC Porto (Portugal) and Shahid Beheshti Univ. (Iran, Islamic Republic of);

R. Amezcua-Correa, Univ. of Bath (United Kingdom); J. P. Carvalho, INESC Porto (Portugal) and Univ. do Porto (Portugal); O. Frazão, INESC Porto (Portugal) and Univ. do Porto (Portugal); P. Caldas, INESC Porto (Portugal), Univ. of Porto (Portugal), and Escola Superior de Tecnologia e Gestão de Viana do Castelo (Portugal); J. L. Santos, INESC Porto (Portugal) and Univ. do Porto (Portugal); F. M. Araújo, INESC Porto (Portugal); H. Latifi, Shahid Beheshti Univ. (Iran, Islamic Republic of); F. Farahi, Univ. of North Carolina at Charlotte (United States); L. A. Ferreira, INESC Porto (Portugal); J. C. Knight, Univ. of Bath (United Kingdom)

7503 3A Micro fiber-optic Fabry-Perot interferometer fabricated by chemical etching of Er-doped fiber [7503-111]

Y. Gong, Y.-J. Rao, Y. Guo, Y. Wu, Z.-L. Ran, Univ. of Electronic Science and Technology of China (China)

7503 3B A fiber-optic liquid sensor for simultaneously measuring refractive index, surface tension, contact angle, and viscosity [7503-112]

A. Zhou, J. Yang, B. Liu, L. Yuan, Harbin Engineering Univ. (China)

7503 3C Sensitivity enhancement of LPG vibration sensor using higher-order cladding mode [7503-113]

S. Tanaka, H. Somatomo, A. Wada, N. Takahashi, National Defense Academy (Japan)

7503 3D Extraordinary pressure sensitivity of long-period gratings written in a boron co-doped optical fiber [7503-114]

M. Smietana, Univ. du Québec en Outaouais (Canada) and Warsaw Univ. of Technology (Poland); W. J. Bock, J. Chen, P. Mikulic, Univ. du Québec en Outaouais (Canada)

7503 3E Fibre laser torsion sensor system using an excessively tilted fibre grating and low-cost time domain demodulation [7503-115]

C. Mou, K. Zhou, R. Suo, L. Zhang, I. Bennion, Aston Univ. (United Kingdom)

7503 3F Non-terminal miniature fiber Bragg grating temperature probe based in U-shape lossless taper [7503-116]

D. Viegas, Univ. do Porto (Portugal) and INESC Porto (Portugal); S. Abad, FiberSensing (Portugal) and Univ. Europea de Madrid (Spain); J. L. Santos, Univ. do Porto (Portugal) and INESC Porto (Portugal); L. A. Ferreira, F. M. Araújo, INESC Porto (Portugal) and FiberSensing (Portugal)

7503 3G A WDM dual sensor system for simultaneous measurement of temperature and pressure [7503-117]

Y. Yang, C. Xu, W. Yang, P. Zhang, BeiHang Univ. (China); W. Jin, The Hong Kong Polytechnic Univ. (Hong Kong, China) 
$75033 \mathrm{H} \quad$ Combined application of Bragg gratings and dielectric sensors for the cure monitoring of bonded composite repairs [7503-118]

G. Tsamasphyros, K. Kalkanis, G. Kanderakis, N. Pantelelis, National Technical Univ. of Athens (Greece); M. Tur, Y. Botsev, N. Gorbatov, Tel Aviv Univ. (Israel); G. Maistros, M. Kazilas, Integrated Aerospace Sciences Corp. (Greece); S. Ronen, U. Ben-Simon, I. Kressel, A. K. Green, Israel Aerospace Industries Ltd. (Israel); R. Chemama, E. Inglisian, GMI-Aero Ltd. (France); F. Voelkermeyer, F. Fischer, Laser Zentrum Hannover e.V. (Germany)

7503 31 Asynchronous FBG interrogation system for temperature and strain monitoring on hydrogenerators rotors [7503-119]

C. Florídia, J. B. Rosolem, F. Borin, E. W. Bezerra, J. C. Said, CpqD Foundation (Brazil)

7503 3J Torsion of air-hole microstructured fiber devices under large defection [7503-120] C. M. Jewart, K. P. Chen, Univ. of Pittsburgh (United States); I. Avdeev, ANSYS Inc. (United States); A. Michie, P. Hambley, J. Canning, Univ. of Sydney (Australia)

7503 3K Temperature independent torsion sensor based on modal interferometry in ultra high-birefringent photonic crystal fiber [7503-121]

O. Frazão, INESC Porto (Portugal) and Univ. do Porto (Portugal); C. Jesus, INESC Porto (Portugal); J. M. Baptista, Univ. da Maderia (Portugal) and INESC Porto (Portugal); J. L. Santos, Univ. do Porto (Portugal) and INESC Porto (Portugal); P. Roy, XLIM, CNRS, Univ. de Limoges (France)

7503 3L High-sensitivity vibration sensing using in-fiber Fabry-Perot interferometer with fiber-Bragg-grating reflectors [7503-122]

A. Wada, S. Tanaka, N. Takahashi, National Defense Academy (Japan)

$75033 \mathrm{M} \quad$ Trial analysis of swine's periodontal ligament with Bragg grating sensors [7503-123] G. F. Menegotto, Federal Univ. of Technology, Paraná (Brazil) and Univ. Positivo (Brazil); L. Grabarski, H. J. Kalinowski, Federal Univ. of Technology, Paraná (Brazil); J. A. Simões, Univ. de Aveiro (Portugal)

$75033 \mathrm{~N}$ Optical-frequency-comb stabilised lasers for interrogation of fibre-resonator strain sensors [7503-124]

G. Gagliardi, M. Salza, P. Ferraro, P. De Natale, Istituto Nazionale di Ottica Applicata, CNR (Italy)

750330 A macrobending fiber based micro-displacement sensor utilizing whispering-gallery modes [7503-125]

P. Wang, Y. Semenova, Q. Wu, G. Farrell, Dublin Institute of Technology (Ireland)

$75033 \mathrm{P} \quad$ Compact in-reflection fiber interferometer using thermally expanded core fiber and single-mode fiber and its applications as temperature and refractive index sensors [7503-126]

L. V. Nguyen, Y. Chung, Gwangju Institute of Science and Technology (Korea, Republic of)

$75033 Q$ Fine-tuning of the transmission characteristics of an arc-induced long-period fiber grating by local heating [7503-127]

D. Monzón-Hernández, I. Torres-Gómez, A. Martínez-Ríos, K. Salas-Alcántara, Cłr. de Investigaciones en Óptica, A.C. (Mexico) 
7503 3R Temperature insensitive accelerometer based on a strain-chirped FBG [7503-128] W. Zhou, X. Dong, K. Ni, China Jiliang Univ. (China); C. C. Chan, P. Shum, Nanyang Technological Univ. (Singapore)

$750335 \quad$ Pressure-gradient fiber laser hydrophone [7503-129]

W. Zhang, F. Zhang, F. Li, Y. Liu, Institute of Semiconductors (China)

7503 3T Simultaneous measurement of liquid level and specific gravity based on fiber Bragg grating sensors [7503-130]

C.-W. Lai, National Cheng Kung Univ. (Taiwan); Y.-L. Lo, National Cheng Kung Univ. (Taiwan) and Feng Chia Univ. (Taiwan); W.-F. Liu, Feng Chia Univ. (Taiwan); J.-P. Yur, Kun Shan Univ. (Taiwan)

7503 3U Displacement measurement by sensor unit with a pair of crossed optical fibers [7503-131] H. Naruse, Y. Yoshikawa, Mie Univ. (Japan); G. Arevalo, J. P. Gonzalez, A. Alvarez, L. Mujica, Micomo (Chile)

7503 3V Fiber grating laser current sensor based on magnetic force [7503-132]

B.-O. Guan, Jinan Univ. (China) and Dalian Univ. of Technology (China); S.-N. Wang, Dalian Univ. of Technology (China)

7503 3W High temperature fiber sensor based on a thermo-mechanical written LPG [7503-133]

J. M. Lazaro, A. Quintela, P. B. Garcia-Allende, J. Mirapeix, C. Galindez, J. M. Lopez-Higuera, Univ. de Cantabria (Spain)

7503 3X 3-axis accelerometer based on lasers locked to $\pi$-shifted fibre Bragg gratings [7503-134] G. Gagliardi, M. Salza, Istituto Nazionale di Ottica Applicata, CNR (Italy); T.-Y. Lam, J. H. Chow, Australian National Univ. (Australia); P. De Natale, Istituto Nazionale di Ottica Applicata, CNR (Italy)

7503 3Y Passive optical sensor for lightning detection on overhead power lines [7503-135] J. B. Rosolem, C. F. Barbosa, C. Floridia, E. L. Bezerra, CPqD Foundation (Brazil)

$75033 Z$ Dual-polarization fiber grating laser displacement sensor [7503-136] Y. Zhang, Dalian Univ. of Technology (China); B.-O. Guan, Dalian Univ. of Technology (China) and Jinan Univ. (China)

750340 Temperature-insensitive 2-D fiber Bragg grating inclinometer [7503-137] S. He, X. Dong, K. Ni, China Jiliang Univ. (China); C. C. Chan, P. Shum, Nanyang Technological Univ. (Singapore)

$750341 \quad$ A miniature extrinsic fiber Fabry-Perot pressure sensor based on fiber etching [7503-138] Y. Ge, M. Wang, C. Yang, Nanjing Normal Univ. (China)

\section{Part Two}

750342 Bending sensing characteristics of long-period gratings UV-point-by-point induced in non-birefringent fibres [7503-139]

R. Z. V. Costa, G. R. C. Possetti, R. Falate, F. A. M. Saccon, M. Muller, J. L. Fabris, Univ. Tecnológica Federal do Paraná (Brazil) 
750343 A temperature self-adapting FBG vibrating sensor system [7503-140]

J.-Y. Wang, Shandong Academy of Sciences (China); D.-H. Huo, Shandong Micro-Sensor Photonics, Ltd. (China); J. Chang, X.-H. Liu, J. Liu, Shandong Academy of Sciences (China); L.-Z. Ma, T.-Y. Liu, Shandong Micro-Sensor Photonics, Ltd. (China); C. Wang, Shandong Academy of Sciences (China)

750344 Interrogation of birefingent fiber sensors using non-reciprocal phase modulation [7503-141] S. Wildermuth, K. Bohnert, H. Brändle, ABB Ltd. (Switzerland)

750345 Optomechanical fiber gyroscope [7503-142] O. Kilic, O. C. Akkaya, H. Ra, M. Digonnet, G. Kino, O. Solgaard, Stanford Univ. (United States)

$750346 \quad$ New methods for modal decomposition in multi-mode fibres [7503-143] S. Blin, T. N. Nguyen, D. M. Nguyen, P. Rochard, Lab. Foton, CNRS, Univ. de Rennes 1 (France); L. Provino, A. Monteville, Perfos (France); T. Robin, iXFiber (France); A. Mugnier, Quantel, Etablissement R\&D Lannion (France); B. Cadier, iXFiber (France); D. Pureur, Quantel, Etablissement R\&D Lannion (France); M. Thual, T. Chartier, Lab. Foton, CNRS, Univ. de Rennes 1 (France)

750347 Experiment on bias stability measurement of resonator fiber optic gyro with digital feedback scheme [7503-144]

X. Wang, Z. He, K. Hotate, The Univ. of Tokyo (Japan)

750348 Performance improvement of PGC method by using lookup table for optical seismometer [7503-145]

C. Tian, L. Wang, M. Zhang, H. Zhang, X. Chu, S. Lai, Y. Liao, Tsinghua Univ. (China)

750349 Effect of fiber tapering in LPG-based Mach-Zehnder modal interferometers for refractive-index sensing [7503-146]

P. Caldas, INESC Porto (Portugal), Univ. do Porto (Portugal), and Escola Superior de Tecnologia e Gestão de Viana do Castelo (Portugal); P. A. S. Jorge, F. Araújo, L. A. Ferreira, INESC Porto (Portugal); G. Rego, INESC Porto (Portugal) and Escola Superior de Tecnologia e Gestão de Viana do Castelo (Portugal); J. L. Santos, INESC Porto (Portugal) and Univ. do Porto (Portugal)

7503 4A Ultra-narrow band transmission filter using a phase-shift chirped fiber Bragg grating concatenated with a broadband rejection filter [7503-147]

D. A. Pereira, Univ. do Porto (Portugal) and Inesc-Porto (Portugal); F. M. Araújo, L. A. Ferreira, INESC-Porto (Portugal); M. B. Marques, J. L. Santos, Univ. do Porto (Portugal) and INESC-Porto (Portugal); J. P. Carmo, ESTEC (Netherlands)

7503 4B Channeled spectral interferometer based fiber optic bending sensor [7503-1 148] L. Yuan, J. Yang, Harbin Engineering Univ. (China)

7503 4C A combined fiber Bragg grating and interferometric based sensor [7503-149] M. Abolbashari, A. S. Gerges, F. Farahi, Univ. of North Carolina at Charlotte (United States) 
7503 4D Novel passive fibre-cavity design for ring-down experiments using a multimode optical waveguide [7503-150]

M. Fabian, E. Lewis, T. Newe, Univ. of Limerick (Ireland); S. Lochmann, Hochschule Wismar (Germany)

7503 4E Realization and tests of an open loop multimode fiber optic gyroscope [7503-151]

H. Medjadba, Lab. of Electronics \& Optoelectronics Systems (Algeria); S. Lecler, ENSPS/UDS

(France); L. M. Simohamed, Lab. of Electronics \& Optoelectronics Systems (Algeria);

A. Chakari, P. Meyrueis, ENSPS/UDS (France)

7503 4F Highly polarizing single-mode optical fiber for sensing applications [7503-152]

C.-H. Wang, B. Jacobsen, D. Bolte, P. Fournier, J. Mayfield, A. Soufiane, Verrillon Inc. (United States)

7503 4G Research on a novel fiber-optic acoustic/rotation sensor array based on the Sagnac interferometer [7503-153]

Z. Song, M. Yang, X. Zhang, C. Cao, S. Xiong, National Univ. of Defense Technology (China)

$75034 \mathrm{H} \quad$ Performance comparison of fiber-optic gyroscopes using single pass backward and double pass backward superfluorescent fiber sources [7503-154]

Y.-W. Huang, T.-S. Peng, L. A. Wang, National Taiwan Univ. (Taiwan); R.-Y. Liu, National Space Organization (Taiwan)

75034 Polarization properties of LPG-based Mach-Zehnder and Michelson intermodal interferometers [7503-155]

T. Eftimov, The Paissi Hilendarski Univ. of Plovdiv (Bulgaria); W. J. Bock, P. Mikulic, J. Chen, Univ. du Québec en Outaouais (Canada)

$75034 \mathrm{~J} \quad$ Fabry-Perot type composite fiber interferometer for gaseous hydrogen leakage detection [7503-156]

K. S. Park, H. Y. Choi, Gwangju Institute of Science and Technology (Korea, Republic of);

K. H. Kwack, Korea Photonics Technology Institute (Korea, Republic of); M.-S. Park,

J.-H. Jang, B. H. Lee, Gwangju Institute of Science and Technology (Korea, Republic of)

7503 4K Long distance (2.1 km) DFB fibre laser hydrophone system [7503-157]

Z. Chen, J. Ng, Institute for Infocomm Research (Singapore)

$75034 \mathrm{~L} \quad$ Field demonstration of a DFB fibre laser hydrophone seabed array in Jervis Bay, Australia [7503-158]

S. Goodman, S. Foster, J. Van Velzen, H. Mendis, Defence Science and Technology

Organisation (Australia)

$75034 \mathrm{M}$ Fiber-optic sensor for real-time monitoring of temperature on high voltage (400KV) power transmission lines [7503-159]

T. K. Gangopadhyay, M. C. Paul, Central Glass and Ceramic Research Institute (India);

L. Bjerkan, SINTEF (Norway)

$75034 \mathrm{~N} \quad$ Fiber optic methane monitor based TDLAS and its application in VAM Oxidiser system [7503-160]

Y. Wei, Y. Li, Y. Zhao, T. Liu, Shandong Academy of Sciences (China) 
$750340 \quad$ Field monitoring of static, dynamic, and statnamic pile loading tests using fibre Bragg grating strain sensors [7503-161]

J. Li, R. Correia, E. Chehura, S. Staines, S. W. James, R. P. Tatam, Cranfield Univ. (United Kingdom); A. P. Butcher, Building Research Establishment (United Kingdom); R. Fuentes, Arup Geotechnics (United Kingdom) and Univ. College London (United Kingdom)

7503 4P Overhead power lines temperature measurements by a fiber optic Raman sensor [7503-162] M. Cirigliano, G. Cattaneo, P. Boffi, A. Barberis, Politecnico di Milano (Italy); U. Perini, G. Pirovano, CESI Ricerca (Italy); M. Martinelli, Politecnico di Milano (Italy)

$75034 Q \quad$ Suppression of cable induced noise in an interferometric sensor system [7503-163] O. H. Waagaard, E. Rønnekleiv, S. Forbord, D. Thingbø, Optoplan AS (Norway)

7503 4R FBG technology applied on container gantry overload warning [7503-164] S. He, W. Li, M. Gao, J. Pan, Wuhan Univ. of Technology (China)

$75034 \mathrm{~S} 250 \mathrm{kHz}$ sampling rate FBG interrogator with strong anti-aliasing signal processing [7503-165] J. Bentell, D. Uwaerts, J. Cloots, T. Bocquet, J. Neys, XenICs NV (Belgium); J. Vlekken, B. Boons, J. Van Roosbroeck, Fibre Optic Sensors and Sensing Systems (Belgium); P. Hong, Tredek Inc (United States)

$75034 \mathrm{~T} \quad$ Welding diagnostics by means of line-to-continuum method and SFFS spectral band selection [7503-166]

J. Mirapeix, P. B. García-Allende, A. Cobo, O. M. Conde, M. Lomer, J. M. López-Higuera, Univ. de Cantabria (Spain)

$75034 \mathrm{U} \quad$ High speed, high performance, portable, dual-channel, optical fiber Bragg grating (FBG) demodulator [7503-167]

H. Zhang, Z. Wei, L. Fan, P. Wang, Stevens Institute of Technology (United States); X. Zhao, Z. Wang, S. Yang, Yantai Univ. (China); H.-L. Cui, Stevens Institute of Technology (United States)

\section{CHEMICAL, ENVIRONMENTAL, BIOLOGICAL, AND MEDICAL SENSORS}

$75034 \mathrm{~W} \quad$ Experimental verification of the effect of slow light on molecular absorption [7503-169] L. Thévenaz, S. Chin, I. Dicaire, J.-C. Beugnot, S. Foaleng Mafang, Ecole Polytechnique Fédérale de Lausanne (Switzerland); M. González Herráez, Univ. de Alcalá (Spain)

7503 4X In vivo 4D imaging of the human lower airway using anatomical optical coherence tomography [7503-170]

R. A. McLaughlin, Univ. of Western Australia (Australia); J. P. Williamson, Sir Charles Gairdner Hospital (Australia) and Univ. of Western Australia (Australia); A. Curatolo, Univ. of Western Australia (Australia); V. A. Baker, D. R. Hillman, Sir Charles Gairdner Hospital (Australia); P. R. Eastwood, Sir Charles Gairdner Hospital (Australia) and Univ. of Western Australia (Australia); D. D. Sampson, Univ. of Western Australia (Australia)

$75034 \mathrm{Y} \quad$ High-speed imaging with endoscopic optical coherence tomography using bending vibration of optical fiber [7503-171]

R. Isago, K. Nakamura, Tokyo Institute of Technology (Japan) 
$75034 Z$ Dynamic analysis of laser ablation of biological tissue by real-time imaging of optical coherence tomography [7503-172]

M. Ohmi, M. Ohnishi, D. Takada, M. Haruna, Osaka Univ. (Japan)

750350 Remote methane sensor using Tunable Diode Laser Spectroscopy (TDLS) via a IW Raman Source [7503-173]

D. Mitchell, K. Duffin, W. Johnstone, Univ. of Strathclyde (United Kingdom)

\section{INTERFEROMETRIC AND POLARIMETRIC SENSORS}

750351 Large interferometers for small displacements: a technological view of gravitational wave detection (Invited Paper) [7503-174]

K. A. Strain, Univ. of Glasgow (United Kingdom)

750352 Fiber laser strain sensors: enabling a new generation of miniaturized high performance sensors [7503-175]

G. A. Cranch, Naval Research Lab. (United States); S. Foster, Defence Science and Technology Lab. (Australia); C. K. Kirkendall, Naval Research Lab. (United States)

750353 Sensitivity improvement of waveguide-type optical passive ring resonator gyroscope by carrier suppression [7503-176]

H. Ma, The Univ. of Tokyo (Japan) and Zhejiang Univ. (China); Z. He, K. Hotate, The Univ. of Tokyo (Japan)

750354 Optical load sensors utilizing fiber-Bragg-grating based Fabry-Perot resonators [7503-177] X. Shu, K. Sugden, I. Bennion, Aston Univ. (United Kingdom)

750355 Linearized interrogation of FDML FBG sensor system using PMF Sagnac interferometer [7503-178]

H. D. Lee, E. J. Jung, M. Y. Jeong, C.-S. Kim, Pusan National Univ. (Korea, Republic of)

750356 Highly versatile in-reflection photonic crystal fibre interferometer [7503-179]

R. Jha, J. Villatoro, M. Kreuzer, V. Finazzi, Instituto de Ciencias Fotónicas (Spain); V. Pruneri, Instituto de Ciencias Fotónicas (Spain) and Institució Catalan de Recerca I Estudi Avançats (Spain)

\section{MICRO AND NANO-ENGINEERED SENSORS AND DEVICES}

750357 Exposed-core microstructured fibres for real-time fluorescence sensing [7503-180] S. C. Warren-Smith, H. Ebendorff-Heidepriem, T. C. Foo, R. Moore, The Univ. of Adelaide (Australia); C. Davis, Defence Science and Technology Organisation (Australia);

T. M. Monro, The Univ. of Adelaide (Australia)

750358 Microstructured chalcogenide fibers for biological and chemical detection: case study: a $\mathrm{CO}_{2}$ sensor [7503-181]

L. Brilland, PERFOS (France); F. Charpentier, J. Troles, B. Bureau, C. Boussard-Plédel,

J. L. Adam, Sciences Chimiques de Rennes, CNRS, Univ. de Rennes 1 (France); D. Méchin,

D. Trégoat, PERFOS (France) 
750359 Miniature fiber-optic temperature sensors based on silica/polymer microfiber knot resonators [7503-182]

Y. WU, Y.-J. Rao, Y. Chen, Univ. of Electronic Science and Technology of China (China)

$75035 \mathrm{~A} \quad$ Ultrasensitive refractive index sensor based on twin-core photonic bandgap fibers [7503-183]

W. Yuan, Technical Univ. of Denmark (Denmark); G. E. Town, Macquarie Univ. (Australia);

O. Bang, Technical Univ. of Denmark (Denmark)

7503 5B Photonic bandgap fiber tapers and interferometric sensors [7503-184]

J. Ju, L. Ma, W. Jin, The Hong Kong Polytechnic Univ. (Hong Kong, China)

7503 5C Gas sensing with suspended core fibres and hollow core band gap fibres: a comparative study [7503-185]

H. Lehmann, J. Kobelke, K. Schuster, R. Willsch, H. Bartelt, IPHT Jena (Germany);

R. Amezcua-Correa, J. C. Knight, Univ. of Bath (United Kingdom)

$75035 \mathrm{D}$ Fibre laser using a microchannel based loss tuning element for refractive index sensing [7503-186]

C. Mou, K. Zhou, E. Davies, L. Zhang, I. Bennion, Aston Univ. (United Kingdom)

POSTERS III

7503 5E Guidelines for the characterization and use of fibre optic sensors: basic definitions and a proposed standard for FBG-based strain sensors [7503-187]

W. R. Habel, Bundesanstalt für Materialforschung und -prüfung (Germany); I. Baumann, AOS GmbH Dresden (Germany); F. Berghmans, Vrije Univ. Brussel (Belgium); K. Borzycki, National Institute of Telecommunications (Poland); C. Chojetzki, FBGS Technologies GmbH (Germany); K.-H. Haase, Hottinger Baldwin Messtechnik GmbH (Germany); L. R. Jaroszewicz, Military Univ. of Technology (Poland); T. Kleckers, Hottinger Baldwin Messtechnik GmbH (Germany); M. Niklès, Omnisens S.A. (Switzerland); M. Rothhardt, Institut für Photonische Technologien Jena e.V. (Germany); V. Schlüter, Bundesanstalt für Materialforschung und -prüfung (Germany); L. Thévenaz, Ecole Polytechnique Fédérale de Lausanne (Switzerland); M. Tur, Tel-Aviv Univ. (Israel); M. Wuilpart, Faculté Polytechnique de Mons (Belgium)

$75035 \mathrm{~F} \quad$ Fiber Bragg grating strain sensor interrogation with $1.3 \mu \mathrm{m}$ Fourier domain mode-locked wavelength swept laser [7503-188]

B. C. Lee, M. H. Oh, M. Y. Jeon, Chungnam National Univ. (Korea, Republic of); E. J. Jung, C.-S. Kim, Pusan National Univ. (Korea, Republic of)

$75035 G \quad$ Bridge external prestressing monitoring based on FBG [7503-189]

S. Li, D. Jiang, Wuhan Univ. of Technology (China)

$75035 \mathrm{H} \quad$ FBG application in bridge health monitoring system of Wuhan Yangtze River 2nd Bridge [7503-190]

J. Liv, Wuhan Univ. of Technology (China) 
$750351 \quad$ Measurement of distributed strain and load identification using $1500 \mathbf{m m}$ gauge length FBG and optical frequency domain reflectometry [7503-191]

H. Igawa, Japan Aerospace Exploration Agency (Japan); H. Murayama, The Univ. of Tokyo (Japan); T. Nakamura, I. Yamaguchi, Japan Aerospace Exploration Agency (Japan);

K. Kageyama, K. Uzawa, D. Wada, I. Onsawa, M. Kanai, The Univ. of Tokyo (Japan);

K. Omichi, Fujikura Ltd. (Japan)

7503 5J Multicomponent strain development in superconducting magnet coils using optical fibre grating sensors fabricated in highly linearly birefringent fibre [7503-192]

E. Chehura, S. W. James, Cranfield Univ. (United Kingdom); A. Twin, F. Domptail, Oxford Instruments NanoScience (United Kingdom); R. P. Tatam, Cranfield Univ. (United Kingdom)

7503 5K Innovative FBG sensing techniques for the railway industry: application to overhead contact line monitoring [7503-193]

G. Laffont, N. Roussel, S. Rougeault, J. Boussoir, L. Maurin, P. Ferdinand, Lab. de Mesures

Optics, CEA (France)

$75035 \mathrm{~L} \quad$ Superimposed long period and short period Bragg grating sensor for LRI monitoring [7503-194]

S. Triollet, Lab. Hubert Curien, CNRS (France) and Univ. of Toulouse (France); L. Robert, Univ. of Toulouse (France); E. Marin, Y. Ouerdane, Lab. Hubert Curien, CNRS (France)

$75035 \mathrm{M} \quad$ Brillouin optical fiber distributed sensor for settlement monitoring while tunneling the metro line 3 in Cairo, Egypt [7503-195]

V. Dewynter, S. Rougeault, S. Magne, P. Ferdinand, Lab. de Mesures Optiques, CEA

(France); F. Vallon, L. Avallone, E. Vacher, M. de Broissia, Bouygues Travaux Publics (France);

C. Canepa, A. Poulain, ACOME (France)

$75035 \mathrm{~N} \quad$ Response of embedded fibre Bragg gratings: strain transfer effects [7503-196]

E. Voet, G. Luyckx, J. Degrieck, Ghent Univ. (Belgium)

750350 Directional bend sensor based on asymmetric long period grating in photonic crystal fiber [7503-197]

L. Jin, W. Jin, J. Ju, The Hong Kong Polytechnic Univ. (Hong Kong, China)

$75035 \mathrm{P}$ Benchmarking the response of Bragg gratings written in micro-structured and bow tie fiber embedded in composites [7503-198]

G. Luyckx, E. Voet, Ghent Univ. (Belgium); T. Geernaert, K. Chah, T. Nasilowski, Vrije Univ. Brussel (Belgium); W. De Waele, Ghent Univ. (Belgium); M. Becker, H. Bartelt, IPHT Jena (Germany); W. Urbanczyk, Wroclaw Univ. of Technology (Poland); J. Wojcik, Maria Curie-Skłodowska Univ. (Poland); J. Degrieck, Ghent Univ. (Belgium); F. Berghmans, H. Thienpont, Vrije Univ. Brussel (Belgium)

$75035 Q \quad$ Fiber-optic Fabry-Perot refractive-index tip sensor based on endlessly photonic crystal fiber [7503-199]

M. Deng, Chongqing Univ. (China); Y. Rao, T. Zhu, Chongqing Univ. (China) and Univ. of Electronic Science \& Technology (China); H. Li, Chongqing Univ. (China) 
$75035 R \quad$ Health monitoring of engine blades by using an in-line fiber-optic F-P strain sensor based on hollow-core photonic crystal fiber [7503-200]

Y. Rao, Chongqing Univ. (China) and Univ. of Electronic Science \& Technology (China);

Y. Fan, H. Li, Chongqing Univ. (China); T. Zhu, Chongqing Univ. (China) and Univ. of Electronic Science \& Technology (China)

$750355 \quad$ Fiber Bragg grating system for continuous large-scale monitoring of convergence in Rossio Tunnel [7503-201]

C. B. Barbosa, FiberSensing S.A. (Portugal); L. A. Ferreira, F. M. Araújo, FiberSensing S.A. (Portugal) and INESC Porto (Portugal); L. Gonçalves, Empresa Portuguesa de Obras

Subterrâneas (Portugal); C. D. Gama, Instituto Superior Técnico (Portugal); R. Malva, Â. Silva, V. Freitas, Rede Ferroviária Nacional EPE (Portugal)

$75035 \mathrm{~T}$ Technical textiles with embedded fibre Bragg grating sensors [7503-202] L. Bilro, Univ. de Aveiro (Portugal); H. Cunha, CITEVE (Portugal); J. L. Pinto, Univ. de Aveiro (Portugal); R. N. Nogueira, Univ. de Santiago (Portugal)

$75035 \mathrm{U}$ A miniature fiber-optic refractive-index sensor based on laser-machined Fabry-Perot interferometer tip [7503-203]

Z. Ran, Univ. of Electronic Science \& Technology (China); Y. Rao, Univ. of Electronic Science \& Technology (China) and Chongqing Univ. (China); J. Zhang, B. Xu, Univ. of Electronic Science \& Technology (China)

$75035 \mathrm{~V} \quad$ Fiber optic pressure sensor based on polarization-maintaining photonic crystal fiber for downhole application [7503-204]

H. Y. Fu, The Hong Kong Polytechnic Univ. (Hong Kong, China); C. Wu, Dalian Univ. of Technology (China); M. L. V. Tse, The Hong Kong Polytechnic Univ. (Hong Kong, China); L. Zhang, Dalian Univ. of Technology (China); H. Y. Tam, The Hong Kong Polytechnic Univ. (Hong Kong, China); B.-O. Guan, Dalian Univ. of Technology (China); C. Lu, P. K. A. Wai, The Hong Kong Polytechnic Univ. (Hong Kong, China)

7503 5W Fluid-filled microstructured optical fibers and switching applications [7503-205] Y. Wang, Univ. of Southampton (United Kingdom), The Hong Kong Polytechnic Univ. (Hong Kong, China), and Institut für Photonische Technologien Jena (Germany); W. Jin, L. Jin, X. Tan, The Hong Kong Polytechnic Univ. (Hong Kong, China); H. Bartelt, W. Ecke, K. Moerl, H. Lehmann, J. Kobelke, K. Schroeder, R. Willsch, M. Rothhardt, L. Shan, S. Brueckner, Institut für Photonische Technologien Jena (Germany); D. J. Richardson, Univ. of Southampton (United Kingdom)

7503 5X Practical sensitive fluorescence sensing with microstructured fibres [7503-206] E. P. Schartner, R. T. White, S. C. Warren-Smith, T. M. Monro, The Univ. of Adelaide (Australia)

$75035 Y$ Optical fibre cavity ring down measurement of refractive index with a microchannel drilled by femtosecond laser [7503-207]

K. Zhou, D. Webb, C. Mou, Aston Univ. (United Kingdom); M. Farries, Gooch \& Housego Torquay Ltd. (United Kingdom); N. Hayes, EvanesCo Ltd. (United Kingdom); I. Bennion, Aston Univ. (United Kingdom)

$75035 Z$ Phase sensitivity of hollow-core photonic bandgap fiber to acoustic pressure [7503-208] M. Pang, W. Jin, Hong Kong Polytechnic Univ. (Hong Kong, China) 
$750361 \quad$ Highly birefringent suspended-core microstructured fibre for sensing application [7503-210] Yu. K. Chamorovskiy, V. P. Gubin, S. K. Morshnev, S. A. Nikitov, Institute of Radio Engineering and Electronics (Russian Federation); O. G. Okhotnikov, Tampere Univ. of Technology (Finland); M. V. Ryabko, N. I. Starostin, I. L. Vorob'ev, Institute of Radio Engineering and Electronics (Russian Federation)

750362 Response of a fibre optic long period grating operating at the phase matching turning point to the deposition of a nanostructured coating [7503-213]

S. W. James, S. M. Topliss, S. C. Cheung, R. P. Tatam, Cranfield Univ. (United Kingdom)

750363 Low-loss fusion splicing of single-mode fiber and a photonic crystal fiber suitable for construction of a patch cord for measurement devices [7503-214]

L. R. Jaroszewicz, M. Murawski, K. Stasiewicz, P. Marć, Military Univ. of Technoogy (Poland)

750364 Photonic crystal fiber pressure sensor [7503-215]

F. C. Fávero, S. M. M. Quintero, V. V. Silva, C. Martelli, A. M. B. Braga, I. C. S. Carvalho,

R. W. A. Llerena, Pontifícia Univ. Católica do Rio de Janeiro (Brazil)

750365 Index-guiding supersquare photonic crystal fibers with single polarization single mode [7503-216]

S. Kim, C.-S. Kee, Gwangju Institute of Science and Technology (Korea, Republic of);

C. G. Lee, Chosun Univ. (Korea, Republic of)

750366 Enhancement of temperature measurement by using photonic bandgap effect [7503-217] Y. F. Zhang, C. C. Chan, J. Sun, Nanyang Technological Univ. (Singapore)

750367 Fiber optic surface plasmon resonance sensors with subwavelength metallic gratings: numerical study [7503-218]

S. Roh, H. Kim, B. Lee, Seoul National Univ. (Korea, Republic of)

750368 Three-dimensional colloidal crystals microstucture fiber: fabrication and characterization [7503-219]

H. Yan, M. Wang, Y. Ge, P. Yu, Nanjing Normal Univ. (China)

750369 Optical sampling technique applied to high resolution distributed fibre sensors [7503-220]

S. Foaleng-Mafang, J.-C. Beugnot, L. Thévenaz, Ecole Polytechnique Fédérale de Lausanne (Switzerland)

7503 6A General analytical model for distributed Brillouin sensors with sub-meter spatial resolution [7503-221]

L. Thévenaz, J.-C. Beugnot, Ecole Polytechnique Fédérale de Lausanne (Switzerland)

7503 6B Single-ended distributed temperature or strain sensor based on stimulated Brillouin scattering [7503-222]

W. Zou, Z. He, K. Hotate, The Univ. of Tokyo (Japan)

7503 6C Simultaneous measurement of strain and temperature by means of polarization division multiplexing optical frequency domain reflectometry [7503-223]

K. Omichi, Y. Terada, A. Sakamoto, S. Hirafune, Fujikura Ltd. (Japan); D. Wada,

H. Murayama, The Univ. of Tokyo (Japan); H. Igawa, Japan Aerospace Exploration Agency (Japan) 
7503 6D Distortion-free Brillouin distributed sensor using RF shaping of pump pulses [7503-224] A. Zornoza, D. Olier, M. Sagues, A. Loayssa, Univ. Publica de Navarra (Spain)

$75036 \mathrm{E} \quad$ Temperature-dependent attenuation characteristics of fibers for distributed temperature sensing in hot geothermal wells [7503-225]

T. Reinsch, J. Henninges, German Research Centre for Geosciences (Germany)

7503 6F Signal-to-noise ratio improvement in Brillouin sensing [7503-226]

N. Linze, W. Li, X. Bao, Univ. of Ottawa (Canada)

$75036 \mathrm{G}$ Distributed perfluorinated POF strain sensor using OTDR and OFDR techniques [7503-227]

S. Liehr, M. Wendt, K. Krebber, BAM Federal Institute for Materials Research and Testing (Germany)

$75036 \mathrm{H} \quad$ Deformation of Brillouin scattered light power spectrum shape by linear strain distribution [7503-228]

K. Yasuda, H. Naruse, Mie Univ. (Japan); C. H. Li, Neubrex Co., Ltd. (Japan); M. Tateda, Chiba Univ. (Japan)

$750361 \quad$ A new model to calculate the Rayleigh scattering profiles in polymer optical fibers for distributed strain sensing [7503-229]

P. Lenke, K. Krebber, BAM Federal Institute for Materials Research and Testing (Germany)

7503 6J Regional selectable distributed sensor based on reflected optical pulse correlation measurement [7503-230]

X. XU, K. Nonaka, Kochi Univ. of Technology (Japan)

$75036 \mathrm{~K}$ Distributed optical fibre sensing of temperature using time-correlated two-photon excited fluorescence [7503-231]

C. J. Dalzell, T. P. J. Han, I. S. Ruddock, Univ. of Strathclyde (United Kingdom)

$75036 \mathrm{~L}$ The two-photon absorption cross-section of ruby and distributed crystal fibre sensing [7503-232]

C. J. Dalzell, T. P. J. Han, I. S. Ruddock, Univ. of Strathclyde (United Kingdom)

$75036 \mathrm{M} \quad$ Multi-point refractometry using tilted fiber Bragg gratings interrogated by a time-domain technique [7503-233]

C. Caucheteur, M. Wuilpart, P. Mégret, Faculté Polytechnique de Mons (Belgium); J. Albert, Carleton Univ. (Canada)

$75036 \mathrm{~N} \quad$ Simultaneous Brillouin backscattering and Raman spontaneous distributed fiber sensor: analytical and numerical approach [7503-234]

L. A. Ribeiro, A. O. Toledo, Instituto de Estudos Avançados (Brazil) and Instituto Nacional de Pesquisas Espaciais (Brazil)

750360 Pipeline monitoring by Brillouin-scattering-based fibre optic distributed strain sensors: pipeline wall thickness detection [7503-235]

G. P. Gu, W. Revie, Natural Resources Canada (Canada); L. Zou, O. Sezerman, Oz Optics Ltd. (Canada) 
75036 P Demonstration of stable Raman fibre laser with asymmetrical laser cavity including broadband chirped FBG [7503-236]

Y.-E. Im, Gwangju Institute of Science and Technology (Korea, Republic of) and Korea Photonics Technology Institute (Korea, Republic of); K. Chun, Gwangju Institute of Science and Technology (Korea, Republic of); H. Kim, S. Hann, Korea Photonics Technology Institute (Korea, Republic of); D.-H. Kim, Doosan Defense and System (Korea, Republic of); Y. Chung, C.-S. Park, Gwangju Institute of Science and Technology (Korea, Republic of)

$75036 Q \quad$ Align-and-shine photolithography [7503-237]

A. Petrušis, J. H. Rector, K. Smith, S. de Man, D. Iannuzzi, Vrije Univ. Amsterdam (Netherlands)

7503 6R Application of $\pi$-shifted short scan interferometry to remote sensing [7503-238]

R. O'Byrne, S. V. Sergeyev, Waterford Institute of Technology (Ireland)

7503 6S Femtosecond laser induced refractive index structures in polymer optical fibre (POF) for sensing [7503-239]

S. J. Liang, P. J. Scully, The Univ. of Manchester (United Kingdom); J. Schille, Univ. of Applied Sciences Mittweida (Germany); J. Vaughan, The Univ. of Manchester (United Kingdom); W. Perrie, Univ. of Liverpool (United Kingdom)

$75036 \mathrm{~T} \quad \mathrm{~N}$-doped-silica-core polarization maintaining fibre for gyros and other sensors for application in space industry [7503-240]

Yu. K. Chamorovskii, O. V. Butov, G. I. Ivanov, A. A. Kolosovskii, V. V. Voloshin, I. L. Vorob'ev, K. M. Golant, Institute of Radio-Engineering and Electronics (Russian Federation)

$75036 \mathrm{U}$ MOEMS accelerometer based on microfiber knot resonator [7503-241]

Y. Wu, Univ. of Electronic Science and Technology of China (China) and Zhejiang Univ.

(China); X. Zeng, Zhejiang Univ. (China); Y.-J. Rao, Univ. of Electronic Science and

Technology of China (China); C.-L. Hou, G.-G. Yang, Zhejiang Univ. (China)

$75036 \mathrm{~V} \quad$ Novel interrogation technique for Tilted Fiber Bragg Gratings sensors based on single wavelength time delay measurements [7503-242]

M. Pisco, Univ. degli Studi del Sannio (Italy); A. Ricciardi, S. Campopiano, Univ. of Naples

Parthenope (Italy); C. Caucheteur, P. Mégret, Faculté Polytechnique de Mons (Belgium);

A. Cutolo, A. Cusano, Univ. degli Studi del Sannio (Italy)

$75036 \mathrm{~W} \quad$ Fiber-optic refractive index sensor based on cladding-mode resonance [7503-243]

F. Pang, H. Liu, N. Chen, Y. Liu, X. Zeng, Z. Chen, T. Wang, Shanghai Univ. (China)

7503 6X Refractive index sensitivity enhancement of $81^{\circ}$ tilted Bragg gratings by cladding etching [7503-244]

E. Davies, P. Saffari, C. Mou, K. Zou, L. Zhang, Aston Univ. (United Kingdom)

$75036 Y \quad$ In-fiber Michelson interferometer based on double-cladding fiber for refractive index sensing [7503-245]

F. Pang, H. Liu, N. Chen, Y. Liu, X. Zeng, Z. Chen, T. Wang, Shanghai Univ. (China)

750362 A tunable high resolution FBG demodulation system using photonic crystal fiber loop mirrors [7503-246]

T. Bakhvalova, Bauman Moscow State Technical Univ. (Russian Federation); G. Rajan,

Y. Semenova, G. Farrell, Dublin Institute of Technology (Ireland) 
750370 The quest for inexpensive, compact, low phase noise laser sources for fiber optic sensing applications [7503-247]

R. E. Bartolo, A. Tveten, C. K. Kirkendall, Naval Research Lab. (United States)

$750371 \quad$ Regeneration of complex Bragg gratings [7503-248]

S. Bandyopadhyay, Central Glass and Ceramic Research Institute (India); J. Canning, The Univ. of Sydney (Australia); P. Biswas, R. Chakraborty, K. Dasgupta, Central Glass and Ceramic Research Institute (India)

750372 Capillary optical fiber linking approach for biosensors [7503-249]

L. Yuan, X. Zhu, T. Zhang, F. Tian, Q. Dai, J. Yang, Harbin Engineering Univ. (China)

750373 Measurement of refractive index profiles variation along a fusion-splicing tapered optical fiber [7503-250]

M.-H. Wan, L. A. Wang, National Taiwan Univ. (Taiwan)

$750374 \quad$ Fiber Bragg grating cladding mode resonance liquid-level sensor [7503-251]

N. Chen, Southeast Univ. (China) and Shanghai Univ. (China); B. Yun, Y. Cui, Southeast Unv. (China)

750375 Photo-annealing effects for erbium doped fiber sources after gamma irradiation tests by using $\mathbf{5 3 2} \mathbf{\mathrm { nm }}$ and $976 \mathrm{~nm}$ lasers [7503-252]

T.-S. Peng, Y.-W. Huang, L. A. Wang, National Taiwan Univ. (Taiwan); R.-Y. Liu, National Space Organization (Taiwan); F.-I. Chou, National Tsing Hua Univ. (Taiwan)

750376 Influence of system configuration on a ratiometric wavelength measurement system [7503-253]

Q. Wu, P. Wang, Y. Semenova, G. Farrell, Dublin Institute of Technology (Ireland)

750377 Innovative spectroscopy of liquids: a fiber optic supercontinuum source and an integrating cavity for scattering-free absorption measurements [7503-254]

A. G. Mignani, Istituto di Fisica Applicata Nello Carrara, CNR (Italy); H. Ottevaere, Vrije Univ. Brussel (Belgium); L. Ciaccheri, Istituto di Fisica Applicata Nello Carrara, CNR (Italy);

H. Thienpont, Vrije Univ. Brussel (Belgium); I. Cacciari, Istituto di Fisica Applicata Nello Carrara, CNR (Italy); O. Parriaux, Lab. Hubert Curien, CNRS, Univ. Jean Monnet (France); M. Johnson, Sagentia Ltd. (United Kingdom)

750378 Realization of 7-cell hollow-core photonic crystal fibers with low loss in the region between $1.4 \mu \mathrm{m}$ and $2.3 \mu \mathrm{m}[7503-255]$

J. K. Lyngsø, Crystal Fibre A/S (Denmark) and DTU Fotonik (Denmark); B. J. Mangan, Univ. of Bath (United Kingdom); C. Jakobsen, Crystal Fibre A/S (Denmark); P. J. Roberts, DTU Fotonik (Denmark)

750379 A simple method for fabricating phase-shifted fibre Bragg gratings with flexible choice of centre wavelength [7503-256]

E. Chehura, S. W. James, R. P. Tatam, Cranfield Univ. (United Kingdom) 
7503 7A Fiber Bragg grating arrays for high resolution manometry [7503-257]

M. Becker, M. Rothhardt, Institut für Photonische Technologien Jena (Germany); S. Voigt, Technische Univ. Chemnitz (Germany); A. Teubner, Clinical Center Chemnitz GmbH (Germany); T. Lüpke, C. Thieroff, Kunststoff-Zentrum in Leipzig GmbH (Germany); C. Chojetzki, FBGS Technologies GmbH (Germany); H. Bartelt, Institut für Photonische Technologien Jena (Germany)

7503 7B Bending and directional characteristics of weakly tilted fiber Bragg grating [7503-258] Y. Miao, Tianjin Univ. (China); B. Liu, J. Liu, Q. Zhao, Nankai Univ. (China)

7503 7C Flat supercontinuum generation pumped by femtosecond pulses in zero-dispersion wavelength of photonic crystal fiber [7503-259]

Y. Guo, Shenzhen Univ. (China) and Taiyuan Univ. of Technology (China); S. Ruan, Y. Yu,

C. Du, P. Yan, Shenzhen Univ. (China)

7503 7D Coupling between a conventional single mode fiber and an annular core fiber by fused biconical taper [7503-260]

C. Guan, L. Yuan, Harbin Engineering Univ. (China)

7503 7E Real-time optical fiber sensor for hydro-alcoholic solutions [7503-261]

E. Fujiwara, R. T. Takeishi, E. Ono, J. S. dos Santos, A. Hase, C. K. Suzuki, The State Univ. of Campinas (Brazil)

$75037 F \quad$ Fibre Bragg gratings in polymer optical fibre at $980 \mathrm{~nm}$ [7503-262]

J. Terblanche, D. Schmieder, J. Meyer, Univ. of Johannesburg (South Africa)

$75037 G \quad$ All Fiber tunable loss filter [7503-263]

J. Mathew, G. Rajan, Y. Semenova, G. Farrell, Dublin Institute of Technology (Ireland)

7503 7H High temperature resistant fiber Bragg grating lasers for sensing applications [7503-264]

B.-O. Guan, Jinan Univ. (China) and Dalian Univ. of Technology; Y. Zhang, H.-J. Wang,

D. Chen, Dalian Univ. of Technology (China); H.-Y. Tam, Hong Kong Polytechnic Univ. (Hong Kong, China)

7503 7I Optimal cladding structure of holey fiber designed for broadband fiber-optic wave plate [7503-265]

Z.-D. Shi, M.-J. Li, J. Yin, J.-Q. Xiang, Shanghai Univ. (China); Lin, X.-N. Shanghai Xinyingyuan

Group Corp. (China)

\section{PASSIVE AND ACTIVE DEVICES FOR PHOTONIC SENSING}

7503 7J Point of care optical device for sepsis diagnosis [7503-266]

F. Baldini, Istituto di Fisica Applicata Nello Carrara, CNR (Italy); L. Bolzoni, Datamed S.r.L. (Italy); A. Giannetti, Istituto di Fisica Applicata Nello Carrara, CNR (Italy); G. Porro, Datamed S.r.L. (Italy); F. Senesi, C. Trono, Istituto di Fisica Applicata Nello Carrara, CNR (Italy)

7503 7K Characterization of $\mathrm{Tm} / \mathrm{Yb}$ doped phosphate microsphere fibre lasers for potential sensor applications [7503-267]

S. Y. Chen, T. Sun, K. T. V. Grattan, City Univ. London (United Kingdom); A. Pal, R. Sen, Central Glass and Ceramic Research Institute (India); C. Sterner, P. Y. Fonjallaz, Acreo AB (Sweden) 
7503 7L Tunable optical delays based on Brillouin dynamic grating in a polarization maintaining fiber [7503-268]

K.-Y. Song, Chung-Ang Univ. (Korea, Republic of); K. Lee, Korea Institute of Science and Technology (Korea, Republic of); H.-J. Yoon, Korea Railroad Research Institute (Korea, Republic of); S. B. Lee, Korea Institute of Science and Technology (Korea, Republic of)

$75037 \mathrm{M} \quad$ Low noise semiconductor laser for optical fiber sensing [7503-269]

M. Poulin, S. Ayotte, A. Babin, M. Aubé, C. Latrasse, J.-F. Cliche, TeraXion Inc. (Canada)

$75037 \mathrm{~N}$ Long-period fiber gratings in twisted single-mode fibers written by high-frequency $\mathrm{CO}_{2}$ laser pulses [7503-270]

T. Zhu, Y.-J. Rao, Chongqing Univ. (China) and Univ. of Electronic Science \& Technology

(China); K. S. Chiang, Chongqing Univ. (China), Univ. of Electronic Science \& Technology

(China), and City Univ. of Hong Kong (Hong Kong, China); C. Shi, M. Liu, Chongqing Univ. (China)

750370 Point-by-point Bragg grating inscription in single-mode microstructure fibre using NIR femtosecond laser [7503-271]

K. Kalli, Cyprus Univ. of Technology (Cyprus); T. Geernaert, Vrije Univ. Brussel (Belgium);

C. Koutsides, Cyprus Univ. of Technology (Cyprus); M. Komodromos, Frederick Univ. (Cyprus); T. Nasilowski, Vrije Univ. Brussel (Belgium); W. Urbanczyk, Wroclow Univ. of Technology (Poland); J. Wojcik, Univ. Maria Curie-Skłodowska (Poland); F. Berghmans, H. Thienpont, Vrije Univ. Brussel (Belgium)

7503 7P High sensitivity FBG sensor made in bismuth oxide fibre with ultrafast radiation [7503-272]

D. Grobnic, R. B. Walker, C. W. Smelser, S. J. Mihailov, Communications Research Ctr. Canada (Canada)

COMMERCIAL SYSTEMS AND FIELD TESTS

$75037 Q \quad$ Installation and test procedures of optical strain gauges for aeronautical applications (Invited Paper) [7503-273]

J. Van Roosbroeck, E. Jacobs, E. Voet, J. Vlekken, Fibre Optic Sensors and Sensing Systems (Belgium)

$75037 R$ Design of fiber optical high temperature sensors for gas turbine monitoring [7503-274] M. Willsch, T. Bosselmann, P. Flohr, R. Kull, Siemens AG (Germany); W. Ecke, I. Latka, D. Fischer, Institut für Photonische Technologien Jena (Germany); T. Thiel, AOS GmbH (Germany)

750375 Study of a progressive failure in soil using BEDS [7503-275]

M. Iten, A. M. Puzrin, D. Hauswirth, Institute for Geotechnical Engineering, ETHZ (Switzerland); S. Foaleng-Mafang, J.-C. Beugnot, L. Thévenaz, Institute of Electrical Engineering, EPFL (Switzerland)

$75037 \mathrm{~T}$ High efficiency TDM/WDM architectures for seismic reservoir monitoring [7503-276] P. Nash, A. Strudley, Stingray Geophysical Ltd. (United Kingdom); R. Crickmore, J. DeFreitas, QinetiQ Ltd. (United Kingdom) 
$75037 \mathrm{U}$ Fibre optic ocean bottom seismic cable system: from innovation to commercial success (Invited Paper) [7503-277]

J. T. Kringlebotn, H. Nakstad, M. Eriksrud, Optoplan AS (Norway)

Author Index 


\title{
Conference Committees
}

\author{
Conference Chair \\ Julian Jones, Heriot-Watt University (United Kingdom) \\ Conference Cochairs
}

Brian Culshaw, University of Strathclyde (United Kingdom)

Wolfgang Ecke, Institut für Photonische Technologien Jena (Germany)

José Miguel López-Higuera, Universidad de Cantabria (Spain)

Reinhardt Willsch, Institut für Photonische Technologien Jena

(Germany)

Local Organising Committee

William MacPherson, Heriot-Watt University (United Kingdom)

Robert Maier, Heriot-Watt University (United Kingdom)

International Steering Committee

Richard O. Claus, Nanosonics Inc. (United States)

Anthony D. Dandridge, Naval Research Laboratory (United States)

Young J. Chung, Gwangju Institute of Science and Technology (Korea, Republic of)

Alexis Mendez, MCH Engineering (United States)

David D. Sampson, University of Western Australia (Australia)

Glen A. Sanders, Honeywell Inc. (United States)

Marc R.-H. Voet, Fiber Optic Sensors and Sensing Systems (Belgium)

Ryozo Yamauchi, Fujikura, Ltd. (Japan)

Technical Programme Committee

Xiaoyi Bao, University of Ottawa (Canada)

Wojtek J. Bock, Université du Quebec à Hull (Canada)

Thomas Bosselmann, Siemens AG (Germany)

John Canning, University of Sydney (Australia)

James H. Cole, Naval Research Laboratory (United States)

Stephen Collins, Victoria University (Australia)

John P. Dakin University of Southampton (United Kingdom)

Michael A. Davis, CiDRA Corporation (United States)

Pierre Ferdinand, CEA LIST (France)

Joseph Friebele, Naval Research Laboratory (United States) 
Juan Hernández Cordero, Universidad nacional Autónoma de México (México)

Daniele Inaudi, Smartec SA (Switzerland)

Hisashi Izumita, Nippon Telegraph and Telephone Corp. (Japan)

Leszek R. Jaroszewicz, Military University of Technology (Poland)

Wei Jin, The Hong Kong Polytechnic University (Hong Kong, China)

Hypolito J. Kalinowski, Universidade Tecnológica Federal do Paraná (Brazil)

Alan Kersey, Illumina Inc. (United States)

Byoungho Lee, Seoul National University (Korea, Republic of)

Robert Lieberman, Intelligent Optical Systems (United States)

Manuel López-Amo, Universidad Pública de Navarra (Spain)

Trevor W. MacDougall, Qorex LLC (United States)

Anna G. Mignani, Istiuto di Fisica Applicata Nello Carrara, CNR (Italy)

Koichiro Miyagi, Yamatake Corporation (Japan)

Kentaro Nakamura, Tokyo Institute of Technology (Japan)

Kyunghwan Oh, Yonsei University (Korea, Republic of)

Aritaka Ohno, Japan Aviation Electronics Industry (Japan)

Yun Jiang Rao, University of Electronic Science and Technology,

Chengdu (China)

Glen A. Sanders, Honeywell Inc. (United States)

Jose L. Santos, Universidade de Porto (Portugal)

Luc Thévenaz, Ecole Polytechnique Fédérale de Lausanne

(Switzerland)

Moishe Tur, Tel Aviv University (Israel)

Akira Wada, Fujikura Ltd. (Japan)

Shinji Yamashita, University of Tokyo (Japan)

Session Chairs

Special Workshop Session on Photonic Crystal Fibres for Sensing I

Brian Culshaw, University of Strathclyde (United Kingdom)

Alexis Mendez, MCH Engineering, LLC (United States)

Special Workshop Session on Photonic Crystal Fibres for Sensing II

Alexis Mendez, MCH Engineering, LLC (United States)

Brian Culshaw, University of Strathclyde (United Kingdom)

Smart Structures and Materials

Wolfgang Ecke, Institut für Photonische Technologien Jena (Germany)

Marc R.-H. Voet, FOS\&S Inc. (Belgium) 
Joint Session S\&A/OFS-20

Ken Grattan, City University (United Kingdom)

Manuel López-Amo, Universidad Pública de Navarra (Spain)

Special Workshop Session on Biophotonic Instrumentation and Sensing I

David Sampson, University of Western Australia (Australia)

Reinhardt Willsch, IPHT Jena (Germany)

Special Workshop Session on Biophotonic Instrumentation and Sensing II

Reinhardt Willsch, Institut für Photonische Technologien Jena (Germany)

David Sampson, University of Western Australia (Australia)

Physical, Mechanical, and Electromagnetic Sensors

Thomas Bosselmann, Siemens AG (Germany)

Stephen Collins, Victoria University (Australia)

Distributed Sensing

Leszek R. Jaroszewicz, Military University of Technology (Poland)

Manuel López-Amo, Universidad Pública de Navarra (Spain)

Multiplexing and Sensor Networking

Pierre Ferdinand, CEA LIST (France)

Anthony Dandridge, Naval Research Laboratory (United States)

Chemical, Environmental, Biological and Medical Sensors

Anna Grazia Mignani, CNR-IFAC (Italy)

Robert Lieberman, Intelligent Optical Systems Inc. (United States)

Interferometric and Polarimetric Sensors

Jose L. Santos, Universidade de Porto (Portugal)

Yun Jiang Rao, University of Electronic Science \& Technology (China)

Micro and Nano-Engineered Sensors and Devices

Hypolito J. Kalinowski, Universidade Tecnológica Federal do Paraná (Brazil)

Luc Thévenaz, Ecole Polytechnique Fédérale de Lausanne

(Switzerland)

Passive and Active Devices for Photonic Sensing

John Canning, University of Sydney (Australia)

Xiaoyi Bao, University of Ottawa (Canada)

Commercial Systems and Field Tests

Wojtek J. Bock, Université du Québec à Hull (Canada)

Ralph Tatam, Cranfield University (United Kingdom) 
Closing OFS-20

Julian Jones, Heriot-Watt University (United Kingdom)

Wojtek J. Bock, Université du Québec à Hull (Canada)

Post Deadline Manuscripts

James Cole, Naval Research Laboratory (United States)

John Dakin, University of Southampton (United Kingdom)

\section{Editors' Note}

All contributed papers in their entirety (i.e. not merely an abstract or extract) were peer reviewed for their significance, innovation, and quality by independent members of the Technical Program Committee, and then rated. 


\section{Introduction}

The International Conference on Optical Fibre Sensors (OFS) was first held in London, in 1983. Since then it has become established as the leading forum for all research into optical fibre and guided wave optical systems for instrumentation, sensing and imaging, and their applications in physical, chemical and biological measurement. The conference is held approximately every eighteen months, rotating between the Americas, Europe, and Asia and the Pacific. OFS returned to the United Kingdom with OFS-20, last held in Glasgow in 1994, and returns again now in 2009 in Edinburgh. The conference will be held in association with the Optics and Photonics Division of the Institute of Physics and in cooperation with SPIE. For the first time, the Institute of Physics's conference on Sensors and their Applications is co-located with OFS.

The conference has now passed its first quarter-century and shows every sign of growing in popularity. Over 250 papers from nearly 40 countries will be presented at OFS-20.

It is interesting to reflect on the factors that give OFS its enduring appeal. There is no doubt that the continuing rapid and exciting evolution of the underlying technological base is one component in continuing to stimulate the research community. In 1983, the only well-developed fibres, optical sources, and detectors were those needed to satisfy the demands of the telecommunications industry of the day, with transmission bandwidths orders of magnitude less than those commonplace now. Today's OFS community has access to optical fibres covering a far greater range of wavelength and with precisely controlled dispersion characteristics; micro-structured components provide even greater versatility and control, for example, through the many forms of available gratings, or entirely different types of 'photonic crystal' fibres. In particular, the Bragg grating has proved to be a remarkably effective and versatile component, and it is now ubiquitous as an easily fabricated and discrete sensing element, ideally suited to wavelength division multiplexing. Sources at almost any wavelength and bandwidth are available, and optical signals are easily amplified within fibres. The potential advantages cited 25 years ago for fibre sensors have now become an engineering reality: most notably sensitivity and specificity, dense multiplexing, and safety and reliability-in fact, multiplexing and long-term reliability are emerging as probably the most important attributes of all. A special feature of optical fibre sensors is their ability to provide distributed sensing, which represents the most extreme case of multiplexing, with some projecting that distributed systems will represent the majority of the market.

Despite the stimulus of new technology, arguably the major reason for the continuing significance and popularity of our subject is its relevance to the important problems of the world today: energy; environment; sustainability; and 
health. Even a cursory glance at the contents of the Proceedings of this and recent OFS conferences shows how the community is contributing to the solution of these critical issues: fibre sensors are actively deployed in the search for new energy supplies, in the generation and distribution of electricity, in energy storage, in efficient use of energy, in monitoring our environment whether natural or engineered, in maintaining the civil infrastructure, in transport systems, and in improving the efficiency of manufacturing. Perhaps some of the most exciting developments are at the interface with the life sciences, where optical fibre sensors are an essential component of the new subject of 'bio-photonics' as a vital tool in research in biochemistry and medicine.

The OFS community is exceptionally heterogeneous. It is not 'owned' by a particular scientific or engineering society, but is stewarded by its own International Steering Committee. Conference participants are highly diverse, and come from a wide range of disciplines in engineering and the physical sciences, and to an increasing extent from life sciences as well. Perhaps it is, above all, the intrinsic interdisciplinarity that keeps the conference relevant and active. After all, none of the really important problems in the world today have solutions lying within a single academic discipline, and thus the highly interdisciplinary community that is OFS is uniquely positioned to contribute.

OFS is only ever as good as the quality of the papers presented, and we are grateful to all of our authors for their excellent contributions. However, we would particularly like to thank our Technical Programme Committee, who over the years has undertaken the onerous task of carefully reading and assessing all the submissions, and has been consistently diligent and reliable in providing the advice on which the programme is built. In the preparation of these Proceedings, we have again benefitted from the capable support of the SPIE, their staff have played a major part in the quality of these volumes.

This is the first occasion on which OFS has been organised by the Institute of Physics. We are grateful to all of the conference office team for their hard work and enthusiasm, and in particular we would like to thank Jenny Bremner for her indefatigable dedication.

Whatever your reasons for joining OFS-20, we are delighted to welcome you to the conference, and to the city of Edinburgh.

\author{
Julian Jones \\ Brian Culshaw \\ Wolfgang Ecke \\ José Miguel López-Higuera \\ Reinhardt Willsch
}

